\title{
Decentralized finite-time adaptive consensus of multiagent systems with fixed and switching network topologies
}

\author{
Zhizhong Tu${ }^{\mathrm{a}, \mathrm{b}}$, Hui $\mathrm{Yu}^{\mathrm{a}, *}$, Xiaohua Xia ${ }^{\mathrm{b}}$ \\ ${ }^{a}$ College of Science, China Three Gorges University, Yichang 443002, China \\ ${ }^{b}$ Centre of New Energy Systems, Department of Electrical, Electronics and Computer \\ Engineering, University of Pretoria, Pretoria 0002, South Africa
}

\begin{abstract}
In this paper, finite-time adaptive consensus problem is investigated for firstorder multiagent systems with unknown nonlinear dynamics. Linearly parameterized method is introduced to model unknown nonlinear dynamics of the systems. By only utilizing the local relative position state information between each agent and its neighbors, decentralized finite-time adaptive consensus algorithms are presented with directed fixed and switching network topologies which satisfy detailed balance condition. Based on classical Lyapunov analysis techniques, both finite-time stability and finite-time parameter convergence are guaranteed by making use of the proposed control algorithms. Finally, the results in Simulations part are presented to validate our main results.
\end{abstract}

Keywords: Multiagent systems, Unknown nonlinear dynamics, Finite-time consensus, Finite-time parameter convergence

\section{Introduction}

The topic of distributed coordinated control of multiple dynamical agents has received extensively attention by many researchers over the past few decades [1-7]. This is not only due to an increasing interest in understanding

${ }^{*}$ Corresponding author.

Email addresses: tuzhizhongsina@163.com (Zhizhong Tu), yuhui@ctgu.edu.cn (Hui Yu), xxia@up.ac.za (Xiaohua Xia) 
thought-provoking animal group behaviors, such as flocking and swarming, but also due to its broad applications in diverse places, such as multi-vehicles rendezvous, attitude alignment, formation control of autonomous robots, unmanned aerial vehicles and so forth. Consensus problem is the fundamental problem in multiagent systems. The essence of it is to construct proper control laws so that all agents can attain a consensus decision value by using the information of each agent and its neighbors. In order to achieve improved cooperative performances for multiagent systems, various works have been done in [8-14], to cite only a few.

In recent years, finite-time consensus problem becomes a research hotspot in multiagent systems. The purpose of it is to construct proper control protocols such that finite-time consensus can be attained. Although asymptotic consensus is enough to satisfy practical demand in engineering in general, finite-time consensus is sometimes more desirable for some engineering applications, such as in some situations where rigid convergence time and high precision must be met. Compared with conventional asymptotic consensus, finite-time consensus reveals numerous advantages, for instance, faster response, higher accuracy, and better robustness and anti-disturbance performance against uncertainties and so forth. On account of these superiorities, several kinds of finite-time consensus protocols have been proposed for firstorder $[15,16]$, second-order $[17,18]$ or high-order [19] multiagent systems.

However, most of existing works focus attention on finite-time consensus algorithms design for multiagent systems without unknown nonlinear dynamics. In [20-23], by employing state feedback or adaptive design methods, finite-time consensus problems are investigated for multiagent systems under the absence of unknown nonlinear dynamics. On the current situation, it is a big challenge to construct decentralized finite-time adaptive control laws for multiagent systems with embedded unknown nonlinear dynamics such that finite-time consensus is attained. In [24], a class of distributed controllers is developed for solving finite-time leaderless consensus problem of nonlinear multiagent systems with parametric uncertainties under an undirected graph. In [25], finite-time consensus problem is solved for a group of high-order agents taking into account unknown nonlinear dynamics under undirected fixed network topology. The finite-time stability is derived by employing the homogeneous Lyapunov function, which is too complicated to find a specific form of it. In addition, the finite-time consensus algorithms designed in this paper, which base on local consensus errors and relative position measurements between each agent and its neighbors, are not purely 
decentralized.

Motivated by the observations mentioned above, we research the finitetime consensus problem of first-order leader-following multiagent systems with unknown nonlinear dynamics under the cases of directed fixed and switching network topologies which satisfy detailed balance condition in this paper. It is assumed that the unknown nonlinear dynamics existed in the systems satisfy linearly parameterized condition. Under some assumptions, we propose decentralized finite-time adaptive control schemes for the systems to attain finite-time consensus, meanwhile, the parameter convergence in finite-time is also guaranteed in both cases of directed fixed and switching network topologies. The finite-time control laws injected in each agent of networks are only dependent on the relative position state information of each agent and its neighbors. With the help of graph theory, classic Lyapunov theory, Hölder's inequality and Barbalat's Lemma, stability results derived in this paper indicate that both finite-time consensus as well as finite-time parameter convergence are attained globally.

The innovation of this paper is reflected as the following three aspects: (1) Purely decentralized finite-time adaptive algorithms for first-order multiagent systems with unknown nonlinear dynamics under the cases of directed fixed and switching network topologies are developed to attain finite-time consensus and finite-time parameter convergence. Based on developed purely decentralized schemes, communication links among agents in the systems are not necessary in this paper. They are greatly improved compared to the algorithms proposed in [25]. (2) Instead of using the complicated homogeneous Lyapunov functions in [25], conventional Lyapunov functions are used for stability analysis. By this way, the proofs of our main results are more concise and understandable compared with these of [25]. (3) Both finite-time consensus and finite-time parameter convergence can be attained for multi-agent networks under the cases of directed fixed and switching network topologies. To assure finite-time consensus as well as finite-time parameter convergence at the same is a challenging work. To the best of our knowledge, few researchers concern this issue, except for the authors of [25].

The configuration of the paper is given as follows. Some preliminaries and formal statement of the problem are presented in Section 2. Our main results are given in Section 3 including decentralized finite-time adaptive consensus control with directed fixed and switching network topologies. Section 4 provides a numerical example to verify the validity of the algorithms proposed in Main Results part under the case of directed switching network topologies. 
The conclusions are drawn in the final section.

\section{Preliminaries and problem statement}

\subsection{Notations}

In this paper, $\|x\|_{1}=\sum_{i=1}^{N}\left|x_{i}\right|,\|x\|_{2}=\left(x^{T} x\right)^{1 / 2}$ denote 1-norm, Euclidean norm of vector $x$, respectively. For $\forall x \in \mathbb{R}^{N}$, it is a basic property that $\|x\|_{2} \leq\|x\|_{1}$. For a symmetric matrix $P \in \mathbb{R}^{N \times N}, \lambda_{\max }(P)$ and $\lambda_{\min }(P)$ are respectively used to denote its maximum and minimum eigenvalues. $I_{l}$ is the $l \times l$ identity matrix. Notation $\operatorname{col}\left(z_{1}, \ldots, z_{n}\right)$ is introduced here to denote column vector of $z_{1}, \ldots, z_{n}$.

\subsection{Graph theory}

The information interaction among agents in multiagent systems can be described by a graph [26]. Thus, it is necessary to introduce this useful tool as follows.

A directed graph is denoted by $\mathscr{G}(\mathscr{V}, \mathscr{E})$ consisting of a node set $\mathscr{V}=$ $\{1,2, \ldots, N\}$ and an edge set $\mathscr{E} \subset \mathscr{V} \times \mathscr{V}$ with ordered pair $(i, j) \in \mathscr{E}$, which indicates that node $i$ can obtain information of node $j$. Notation $\mathscr{N}_{i}=\{j \in \mathscr{V} \mid(i, j) \in \mathscr{E}, j \neq i\}$ is introduced to denote the set of neighbors of node $i$. A path in a directed graph is a sequence of distinct edges in $\mathscr{E}$, which connect end to end. If a path exists between any two distinct nodes of a directed graph, this graph is said to be strongly connected. The $i j$ th item of weighted adjacency matrix $\mathscr{A} \in \mathbb{R}^{N \times N}$ of graph $\mathscr{G}$ is denoted as $a_{i j}$, which is positive if $(i, j)$ is an edge of graph $\mathscr{G}$ and 0 otherwise. The degree matrix $\mathscr{D} \in \mathbb{R}^{N \times N}$ of graph $\mathscr{G}$ is a diagonal matrix with diagonal elements $d_{i}=\sum_{j \in \mathscr{N}_{i}} a_{i j}$ for $i \in \mathscr{V}$. And the Laplacian matrix of weighted directed graph $\mathscr{G}$ is expressed as $\mathscr{L}=\mathscr{D}-\mathscr{A}$.

Another graph $\overline{\mathscr{G}}$ on node set $\overline{\mathscr{V}}=\{0,1,2, \ldots, N\}$ is introduced here to represent the information exchange between $N$ follower agents and the leader agent labeled as node 0 , and its edges including $\mathscr{E}$ as well as the edges between leader agent and follower agents. The connection weight between agent $i \in \mathscr{V}$, and leader agent is denoted as $b_{i} \geq 0$, which is positive when leader agent is a neighbor of agent $i$ and 0 otherwise.

A graph is said to be detailed balanced if there exist some real numbers $\omega_{i}>0, i \in \mathscr{V}$, such that the coupling weights of the graph satisfy $\omega_{i} a_{i j}=$ $\omega_{j} a_{j i}$ for all $i, j \in \mathscr{V}[27]$. 
Assumption 1. The directed graph $\mathscr{G}$ is strongly connected and detailed balanced, and $b_{i}>0$ for at least one $i \in \mathscr{V}$.

Remark 1. From Assumption 1, the leader agent is globally reachable. In addition, we only extend the case of undirected graphs slightly to that of detailed balance graphs due to the symmetric requirement in designing purely decentralized parameter adaptive laws. It is a challenging work to consider the case of directed graphs excluding detailed balance graphs. We will consider this case in our future work.

Let $H=\mathscr{L}+\mathscr{B}, \mathscr{B}=\operatorname{diag}\left(b_{1}, b_{2}, \ldots, b_{N}\right), \operatorname{diag}\{\omega\}=\operatorname{diag}\left\{\omega_{1}, \omega_{2}, \ldots, \omega_{N}\right\}$. Then, we have the following lemma based on Lemma 4.1 in [28].

Lemma 1. Under Assumption 1, $\operatorname{diag}\{\omega\} H$ is symmetric and positive definite.

Remark 2. According to the definition of detailed balance graph, it is easy to verify that $\operatorname{diag}\{\omega\} H=H^{T} \operatorname{diag}\{\omega\}$ is symmetric. Based on Lemma 4.1 in [28], we can obtain that $\operatorname{diag}\{\omega\} H$ is positive definite.

\subsection{Problem statement}

The dynamics of $N$ follower agents considered in this paper can be modeled as the following form

$$
\dot{x}_{i}(t)=f_{i}\left(x_{i}(t), t\right)+u_{i}(t), \quad i \in \mathscr{V},
$$

with $x_{i} \in \mathbb{R}$ denoting the $i$ th follower agent's position state, $u_{i} \in \mathbb{R}$ denoting the $i$ th follower agent's control input, and smooth function $f_{i}\left(x_{i}(t), t\right)$ denoting the $i$ th follower agent's unknown nonlinear dynamics. We assume that $f_{i}\left(x_{i}(t), t\right), i \in \mathscr{V}$, are continuous in $t$ and Lipschitz in $x_{i}(t)$ so as to guarantee there exists unique solutions. The leader agent's underlying dynamics considered in this paper can be modeled as the following form

$$
\dot{x}_{0}(t)=v_{0}(t),
$$

with $x_{0} \in \mathbb{R}$ denoting position state of the leader agent, and $v_{0} \in \mathbb{R}$ denoting unknown control input of the leader agent.

Remark 3. In this paper, it is assumed that the states of all agents are scalars in $\mathbb{R}$ to avoid complicated expressions, which can be easily extended to $\mathbb{R}^{n}$ by using the Kronecker product. 
For the sake of obtaining our main results, a useful lemma [29] is introduced here.

Lemma 2. Consider a dynamic system $\dot{x}(t)=f(x(t)), f(0)=0, x \in \mathbb{R}^{N}$, $x(0)=x_{0}$. Suppose that there exists a continuous function $V(x): \mathbb{D} \rightarrow$ $\mathbb{R}$ such that the following conditions hold: (a) $V(x)$ is a positive-definite function; (b) There exist real numbers $c_{\alpha}>0$ and $\alpha \in(0,1)$, and an open neighborhood $\mathbb{H} \subset \mathbb{D}$ of the origin such that $\dot{V}(x) \leq-c_{\alpha} V(x)^{\alpha}, x \in \mathbb{H} \backslash\{0\}$. Then the origin is a finite-time stable equilibrium of this system. Moreover, the settling time $T$ of this system satisfies $T \leq \frac{1}{c_{\alpha}(1-\alpha)} V(x)^{1-\alpha}$.

We assume that the unknown nonlinear dynamics $f_{i}\left(x_{i}(t), t\right), i \in \mathscr{V}$, are parameterized as follows

$$
f_{i}\left(x_{i}(t), t\right)=\phi_{i}^{T}\left(x_{i}(t), t\right) \theta_{i}
$$

and the unknown control input signal of the leader agent is parameterized as follows

$$
v_{0}(t)=\phi_{0}^{T}(t) \theta_{0}
$$

with $\phi_{i}\left(x_{i}(t), t\right), \phi_{0}(t) \in \mathbb{R}^{m}$ denoting basis function column vectors and $\theta_{i}, \theta_{0} \in \mathbb{R}^{m}$ denoting constant true parameter column vectors, which are unknown and should be estimated.

As $\theta_{0}$ is not commonly attainable to each agent, follower agent $i$ estimates leader agent's unknown parameter vectors $\theta_{0}$ by $\hat{\theta}_{0 i}$ and $v_{0}(t)$ by $\hat{v}_{0 i}(t)$ respectively so as to design decentralized controllers. So one has

$$
\hat{v}_{0 i}(t)=\phi_{0}^{T}(t) \hat{\theta}_{0 i}, \quad i \in \mathscr{V} .
$$

And the function $f_{i}\left(x_{i}(t), t\right)$ can be similarly estimated as

$$
\hat{f}_{i}\left(x_{i}(t), t\right)=\phi_{i}^{T}\left(x_{i}(t), t\right) \hat{\theta}_{i}, \quad i \in \mathscr{V} .
$$

Remark 4. In this paper, linearly parameterized method is used to estimate all the unknown nonlinear dynamics of the system. The readers can refer to $[13,25,30-32]$ for more applications of this method. Of course, there are some other feasible methods to handle unknown nonlinear dynamics when finite-time consensus problems of the multiagent systems are considered. The most widely used methods are to assume all agents's unknown nonlinear dynamics satisfy Lipschitz-type condition [33, 34] or bounded condition [35]. 
When the multiagent systems are subject to external disturbances, linearly parameterized method is also feasible to deal with external disturbances if external disturbances of the systems satisfy linearly parameterized assumption. The other approach is to assume disturbances of all agents satisfy bounded condition $[21,36]$.

Remark 5. There are some practical systems satisfying agent's dynamics (1). When the states of all agents are within the scope of $\mathbb{R}^{3}$, the multiagent systems could be unmanned aerial vehicle systems [37] while the states of all agents are within the scope of $\mathbb{R}^{2}$, the multiagent systems could be unmanned vehicle systems in the plane. Terms $u_{i}(t)$ and $f_{i}\left(x_{i}(t), t\right)$ in dynamic equation (1) are respectively the force linear input, and inherently nonlinear input which satisfies linear parameterizations assumption, of agent $i$ in the latter situation (see Fig. 1).

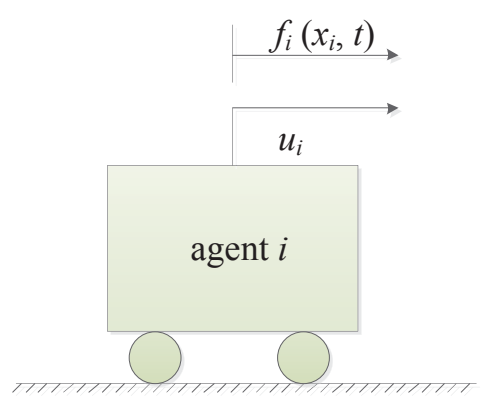

Figure 1: Unmanned vehicle.

Before continuing, a vital assumption should be made to assure parameter convergence in finite-time, that is, the regressor matrix $\Phi$ must satisfy the following peresistently exiciting (PE) [31] condition.

PE condition: Two positive real numbers $\delta_{0}$ and $l_{0}$ exist here to ensure that the following inequality holds:

$$
\int_{t}^{t+\delta_{0}} \Phi \Phi^{T} d \tau \geq l_{0} I>0, \quad \forall t \geq 0 .
$$

Remark 6. As a standard assumption in classical adaptive control [30, 31], the $\mathrm{PE}$ condition is very helpful to ensure parameter convergence and the 
richness of the regressor matrix $\Phi$ information throughout the system time. For more typical applications of $\mathrm{PE}$ condition in multiagent systems, the readers can refer to $[13,25,32]$.

The control purpose in this paper is to design effective decentralized finitetime adaptive consensus schemes to obtain leader-following finite-time consensus while ensuring finite-time parameter convergence, namely

$$
\lim _{t \rightarrow T_{s}}\left\|x_{i}(t)-x_{0}(t)\right\|=0
$$

and

$$
\lim _{t \rightarrow T_{s}}\left\|\hat{\theta}_{0 i}-\theta_{0}\right\|=0, \lim _{t \rightarrow T_{s}}\left\|\hat{\theta}_{i}-\theta_{i}\right\|=0,
$$

with $x_{i}(0)$ being any initial condition of $x_{i}(t), i \in \overline{\mathcal{V}}, T_{s}>0$ being the settling time.

\section{Main results}

3.1. Decentralized finite-time adaptive consensus with directed fixed topology

Decentralized finite-time adaptive consensus problem for the leader-following multiagent system (1)-(2), whose network topology is directed and fixed while satisfying detailed balance condition, will be studied in this part.

For directed fixed network topology, decentralized finite-time adaptive consensus control schemes proposed here consist of the following two parts.

Decentralized finite-time feedback laws:

$$
\begin{aligned}
u_{i}= & -k\left[\omega_{i}\left[\sum_{j \in \mathscr{N}_{i}} a_{i j}\left(x_{i}-x_{j}\right)+b_{i}\left(x_{i}-x_{0}\right)\right]\right]^{\alpha} \\
& +\Phi_{i}^{T} \hat{\Theta}_{i},
\end{aligned}
$$

and decentralized finite-time parameter adaptive laws:

$$
\begin{aligned}
\dot{\hat{\theta}}_{0 i}= & -\frac{k_{1}}{k} \phi_{0}(t) \omega_{i} \times \\
& {\left[\sum_{j \in \mathscr{N}_{i}} a_{i j}\left(x_{i}-x_{j}\right)+b_{i}\left(x_{i}-x_{0}\right)\right], } \\
\dot{\hat{\theta}}_{i}= & \frac{k_{2}}{k} \phi_{i}\left(x_{i}, t\right) \omega_{i} \times \\
& {\left[\sum_{j \in \mathscr{N}_{i}} a_{i j}\left(x_{i}-x_{j}\right)+b_{i}\left(x_{i}-x_{0}\right)\right], }
\end{aligned}
$$


with $\Phi_{i}=\operatorname{col}\left(\phi_{0},-\phi_{i}\right), \hat{\Theta}_{i}=\operatorname{col}\left(\hat{\theta}_{0 i}, \hat{\theta}_{i}\right), \omega_{i}>0, i \in \mathscr{V}, \alpha \in(0,1), k>1$, $k_{1}, k_{2}$ being positive constants. Let $y_{i}=\omega_{i}\left[\sum_{j \in \mathscr{N}_{i}} a_{i j}\left(x_{i}-x_{j}\right)+b_{i}\left(x_{i}-x_{0}\right)\right]$, $\left\lceil y_{i}\right\rfloor^{\alpha}=\left|y_{i}\right|^{\alpha} \operatorname{sgn}\left(y_{i}\right), i \in \mathscr{V}$, where $\operatorname{sgn}(\cdot)$ is the sign function.

Remark 7. In [25], based on local consensus errors and relative position measurements between each agent and its neighbors, the authors presented a finite-time adaptive consensus algorithm for a multiagent system. In fact, it is not purely decentralized. In this regard, purely decentralized laws (10) and (11), which only depend on the local relative position state information between each agent and its neighbors, are proposed here for the systems under the case of fixed network topology. Unlike [25], communication links among agents in the systems are not necessary in terms of purely decentralized laws (10) and (11) in this paper. And these information can be measured by the on-board sensor of each agent, such as ultrasonic or infrared-based relative positioning sensors [38].

Letting $u=\operatorname{col}\left(u_{1}, u_{2}, \ldots, u_{N}\right), x=\operatorname{col}\left(x_{1}, x_{2}, \ldots, x_{N}\right), \bar{x}(t)=x(t)-\mathbf{1}_{N} \otimes$ $x_{0}(t), M=\operatorname{diag}\{\omega\} H, \zeta=\operatorname{col}\left(\zeta_{1}, \zeta_{2}, \ldots, \zeta_{N}\right)=M \bar{x},\left\lceil\zeta_{i}\right\rfloor^{\alpha}=\left|\zeta_{i}\right|^{\alpha} \operatorname{sgn}\left(\zeta_{i}\right)$, $i \in \mathscr{V},\lceil\zeta\rfloor^{\alpha}=\operatorname{col}\left(\left\lceil\zeta_{1}\right\rfloor^{\alpha},\left\lceil\zeta_{2}\right\rfloor^{\alpha}, \ldots,\left\lceil\zeta_{N}\right\rfloor^{\alpha}\right), \Phi=\operatorname{diag}\left\{\Phi_{1}, \Phi_{2}, \ldots, \Phi_{N}\right\}, \hat{\Theta}=$ $\operatorname{col}\left(\hat{\Theta}_{1}, \hat{\Theta}_{2}, \ldots, \hat{\Theta}_{N}\right)$, we have

$$
u=-k\lceil\zeta\rfloor^{\alpha}+\Phi^{T} \hat{\Theta} .
$$

With (12), letting $f=\operatorname{col}\left(f_{1}, f_{2}, \ldots, f_{N}\right), \Theta_{i}=\operatorname{col}\left(\theta_{0}, \theta_{i}\right), \Theta=\operatorname{col}\left(\Theta_{1}, \Theta_{2}\right.$, $\left.\ldots, \Theta_{N}\right), \bar{\Theta}=\hat{\Theta}-\Theta$, and noting that $\dot{x}(t)=f+u$, the error system can be written as follows:

$$
\dot{\bar{x}}(t)=-k\lceil\zeta\rfloor^{\alpha}+\Phi^{T} \bar{\Theta},
$$

and

$$
\dot{\bar{\Theta}}=-\frac{1}{k}\left(I_{N} \otimes K\right) \Phi M \bar{x},
$$

where $K=\left(\begin{array}{cc}k_{1} I_{m} & \mathbf{0} \\ \mathbf{0} & k_{2} I_{m}\end{array}\right)$.

For the system (1)-(2) under the case of directed fixed network topology, the following main theorem can be obtained.

Theorem 1. Consider the multiagent system (1)-(2). Suppose that Assumption 1 holds, $\phi_{i}(t), i \in \overline{\mathcal{V}}$, are uniformly bounded and continuous, and the $P E$ 
condition given in $(7)$ is met. Then, there exists $\varepsilon_{1} \in(0,1)$, such that, for every $\alpha \in\left(1-\varepsilon_{1}, 1\right)$, under feedback laws (10) and parameter adaptive laws (11), we have: (a) global consensus for this system is obtained in finite-time; (b) global parameter convergence is assured in finite-time; (c) the settling time $T_{s}<\frac{2^{(3-\alpha) / 2} N^{\alpha} \lambda_{\max }^{(1+\alpha) / 2}(M)}{(1-\alpha) k^{(1+\alpha) / 2} \lambda_{\min }^{1+\alpha}(M)}$.

For purpose of proving Theorem 1, the following two lemmas are necessary to be proved first.

Before going on, the following Lyapunov candidate function is constructed.

$$
V(t)=\frac{1}{2 k} \bar{x}^{T} M \bar{x} .
$$

Let $\Omega_{\sigma} \triangleq\{\bar{x}: V(t) \leq \sigma\}$.

Lemma 3. Consider the error system (13)-(14). If Assumption 1 holds, there exists $\varepsilon_{1} \in(0,1)$, such that, for every $\alpha \in\left(1-\varepsilon_{1}, 1\right)$, one has: (a) this system is uniformly stable; (b) for $\forall t \geq t_{0}, \forall \bar{x}\left(t_{0}\right) \in \mathbb{R}^{N}, \bar{\Theta}\left(t_{0}\right) \in \mathbb{R}^{2 N m}$, the solution $(\bar{x}, \bar{\Theta})$ of this system is uniformly bounded; (c) for $\forall t \geq t_{0}, \forall \bar{x}\left(t_{0}\right) \in \mathbb{R}^{N}$, $\bar{\Theta}\left(t_{0}\right) \in \mathbb{R}^{2 N m}, \lim _{t \rightarrow \infty}\|\bar{x}(t)\|=0 ;(\mathrm{d})$ there exists $t_{1}^{*}$ such that $\bar{x}(t) \in \Omega_{1}$ for any $t \geq t_{1}^{*}$.

Proof. Lyapunov candidate function is selected as follows:

$$
W(t)=\frac{1}{2 k} \bar{x}^{T} M \bar{x}+\frac{1}{2} \bar{\Theta}^{T}\left(I_{N} \otimes K^{-1}\right) \bar{\Theta} .
$$

Taking the time derivative of $W$ along the solution of the system (13)-(14) yields

$$
\dot{W}=-\zeta^{T}\lceil\zeta\rfloor^{\alpha}
$$

For (17), we use the same method as Proposition 1 in [39]. By selecting $p=1+\alpha, q=(1+\alpha) / \alpha$ in Hölder's inequality, we have

$$
\begin{aligned}
\|\zeta\|_{1} & =\sum_{i=1}^{N}\left(\left|\zeta_{i}\right| \times 1\right) \\
& \leq\left(\sum_{i=1}^{N}\left|\zeta_{i}\right|^{1+\alpha}\right)^{\frac{1}{1+\alpha}}\left(\sum_{i=1}^{N} 1^{\frac{1+\alpha}{\alpha}}\right)^{\frac{\alpha}{1+\alpha}} \\
& =N^{\frac{\alpha}{1+\alpha}}\left(\zeta^{T}\lceil\zeta\rfloor^{\alpha}\right)^{\frac{1}{1+\alpha}} .
\end{aligned}
$$


Applying the above inequality follows that $\zeta^{T}\lceil\zeta\rfloor^{\alpha} \geq N^{-\alpha}\|\zeta\|_{1}^{1+\alpha}$. Noting that $\zeta=M \bar{x}$, we have

$$
\begin{aligned}
\dot{W} & \leq-N^{-\alpha}\|\zeta\|_{1}^{1+\alpha} \\
& \leq-N^{-\alpha}\|\zeta\|_{2}^{1+\alpha} \\
& \leq-N^{-\alpha} \lambda_{\min }^{1+\alpha}(M)\|\bar{x}\|_{2}^{1+\alpha} .
\end{aligned}
$$

Applying the same arguments as in [31], (a) and (b) hold.

From (18), we have $\lim _{t \rightarrow \infty} W(t)=W(\infty)$ and

$$
\frac{\lambda_{\min }^{1+\alpha}(M)}{N^{\alpha}} \lim _{t \rightarrow \infty} \int_{t_{0}}^{t}\|\bar{x}(\tau)\|_{2}^{1+\alpha} d \tau \leq W\left(t_{0}\right)-W(\infty) .
$$

With Barbalat's Lemma [31], it follows that $\lim _{t \rightarrow \infty}\|\bar{x}(t)\|_{2}^{1+\alpha}=0$, which means that (c) holds.

From sign-preserving theorem of continuous function, it follows from (18) that

$$
\lim _{\alpha \rightarrow 1} \dot{W} \leq-\frac{\lambda_{\min }^{2}(M)}{N} \bar{x}^{T} \bar{x}
$$

Thus, there exists $\varepsilon_{1} \in(0,1)$ such that, for every $\alpha \in\left(1-\varepsilon_{1}, 1\right)$, we have

$$
\dot{W} \leq-\frac{\lambda_{\min }^{2}(M)}{N} \bar{x}^{T} \bar{x}
$$

When $\bar{x}(t)$ is outside the set $\Omega_{1}$, from the definition of $\Omega_{1}$, we have $1<$ $\frac{1}{2 k} \bar{x}^{T} M \bar{x} \leq \frac{\lambda_{\max }(M)}{2 k} \bar{x}^{T} \bar{x}$. Then, it follows that

$$
\bar{x}^{T} \bar{x}>\frac{2 k}{\lambda_{\max }(M)} .
$$

Integrating (19) with respect to time $t$, we have $W(t)-W\left(t_{0}\right) \leq-\frac{\lambda_{\min }^{2}(M)}{N} \int_{t_{0}}^{t} \bar{x}^{T} \bar{x} d \tau$. From (16) and (20), it gives that

$$
V(t) \leq W(t)<W\left(t_{0}\right)-l_{1}\left(t-t_{0}\right), \quad \bar{x} \in \mathbb{R}^{N} \backslash \Omega_{1},
$$

where $l_{1}=\frac{2 k \lambda_{\min }^{2}(M)}{N \lambda_{\max }(M)}$. From $(21)$, there exists $t_{1}^{*}=t_{0}+\frac{W\left(t_{0}\right)-1}{l_{1}}$ such that

$$
V(t) \leq W(t)<W\left(t_{0}\right)-l_{1}\left(t-t_{0}\right) \leq 1
$$


as $t \geq t_{1}^{*}$, namely, $\bar{x} \in \Omega_{1}$ as $t \geq t_{1}^{*}$.

Therefore, there exists $t_{1}^{*} \geq t_{0}$,

$$
t_{1}^{*}= \begin{cases}t_{0}+\frac{W\left(t_{0}\right)-1}{l_{1}}, & \text { if } W\left(t_{0}\right)>1 \\ t_{0}, & \text { otherwise }\end{cases}
$$

so that $\bar{x} \in \Omega_{1}$ as $t \geq t_{1}^{*}$. From (16) and (22), we also have

$$
\bar{\Theta}^{T} \bar{\Theta} \leq 2 \max \left\{k_{1}, k_{2}\right\}, \quad t \geq t_{1}^{*} .
$$

This finishes the proof of $(\mathrm{d})$.

Lemma 4. Consider the error system (13)-(14). Suppose that Assumption 1 holds, $\phi_{i}(t), i \in \overline{\mathcal{V}}$, are uniformly bounded and continuous, and the PE condition given in $(7)$ is met. Then, for every $\alpha \in\left(1-\varepsilon_{1}, 1\right)$, under feedback laws (10) and parameter adaptive laws (11), this system is finite-time stable on set $\Omega_{1}$.

Proof. Taking the time derivative of $V$ along the solution of the system (13)-(14), it follows that

$$
\dot{V}=-\zeta^{T}\lceil\zeta\rfloor^{\alpha}+\frac{1}{k} \bar{x}^{T} M \Phi^{T} \bar{\Theta} .
$$

Applying the same method as in Lemma 3, and noting that $V \leq \frac{1}{2 k} \lambda_{\max }(M) \bar{x}^{T} \bar{x}$, we have

$$
\dot{V} \leq-l_{2} V^{\frac{1+\alpha}{2}}+\frac{1}{k} \bar{x}^{T} M \Phi^{T} \bar{\Theta}
$$

where $l_{2}=\frac{(2 k)^{(1+\alpha) / 2} \lambda_{\min }^{1+\alpha}(M)}{N^{\alpha} \lambda_{\max }^{(1+\alpha) / 2}(M)}$.

From Lemma 3 , there exists $t_{1}^{*}$ such that $\bar{x} \in \Omega_{1}$ for any $t \geq t_{1}^{*}$. Denote the non-trivial solution of (13) as $\bar{x}\left(t, t_{0}, \bar{x}\left(t_{0}\right)\right)$. Three different cases will be analyzed as follows:

Case 1 : There exists a time interval $\left[t_{1}, t_{2}\right] \subset\left[t_{1}^{*}, t_{1}^{*}+T_{1}\right]$ such that $\bar{x}\left(t, t_{0}, \bar{x}\left(t_{0}\right)\right)=0$ for any $t \in\left[t_{1}, t_{2}\right]$ with $t_{2}>t_{1}, T_{1}=\frac{2^{(3-\alpha) / 2} N^{\alpha} \lambda_{\max }^{(1+\alpha) / 2}(M)}{(1-\alpha) k^{(1+\alpha) / 2} \lambda_{\min }^{1+\alpha}(M)}$.

In this case, it is obvious that $\bar{x}(t) \equiv 0$ for any $t>t_{1}$. From (14), we obtain $\dot{\bar{\Theta}} \equiv 0$ for any $t>t_{1}$. Then, for any $t>t_{1}$, it can be obtained that $\bar{\Theta}$ is a constant vector. 
For any $t>t_{1}$, we show that $\bar{\Theta} \equiv 0$ by a contradiction method. Define function

$$
\Xi(\bar{\Theta}(t), t)=\frac{1}{2}\left[\bar{\Theta}^{T}\left(t+T_{2}\right) \bar{\Theta}\left(t+T_{2}\right)-\bar{\Theta}^{T}(t) \bar{\Theta}(t)\right],
$$

where $T_{2}>0$.

For $\forall t>t_{1}$, it is obvious that $\Xi(\bar{\Theta}(t), t) \equiv 0$ as $\bar{\Theta}(t)$ is a constant vector, then $\frac{d \Xi}{d t} \equiv 0$. Supposing $\bar{\Theta}(t) \neq 0$ and taking the time derivative of $\Xi(\bar{\Theta}(t), t)$, we have

$$
\begin{aligned}
\frac{d \Xi(\bar{\Theta}(t), t)}{d t} & =\bar{\Theta}^{T}\left(t+T_{2}\right) \dot{\bar{\Theta}}\left(t+T_{2}\right)-\bar{\Theta}^{T}(t) \dot{\bar{\Theta}}(t) \\
& =\int_{t}^{t+T_{2}} \frac{d}{d \tau}\left(\bar{\Theta}^{T}(\tau) \dot{\bar{\Theta}}(\tau)\right) d \tau \\
& =-\frac{1}{k} \int_{t}^{t+T_{2}} \frac{d}{d \tau}\left[\bar{\Theta}^{T}\left(I_{N} \otimes K\right) \Phi M \bar{x}\right] d \tau \\
& \leq-\frac{\min \left\{k_{1}, k_{2}\right\}}{k} \int_{t}^{t+T_{2}} \bar{\Theta}^{T} \Phi M \Phi^{T} \bar{\Theta} d \tau \\
& \leq-l_{3} \int_{t}^{t+T_{2}} \bar{\Theta}^{T} \Phi \Phi^{T} \bar{\Theta} d \tau \\
& =-l_{3} \bar{\Theta}^{T}\left(\int_{t}^{t+T_{2}} \Phi \Phi^{T} d \tau\right) \bar{\Theta}
\end{aligned}
$$

where $l_{3}=\frac{\min \left\{k_{1}, k_{2}\right\} \lambda_{\min }(M)}{k}$. Applying the PE condition defined in (7), (27) becomes

$$
\frac{d \Xi(\bar{\Theta}(t), t)}{d t} \leq-l_{0} l_{3}\|\bar{\Theta}\|_{2}^{2}<0, \quad \forall t>t_{1}
$$

which contradicts that $\frac{d \Xi}{d t} \equiv 0, \forall t>t_{1}$. Hence, for $\forall t>t_{1}, \bar{\Theta} \equiv 0$. Therefore, this error system is finite-time stable under this case.

Case $2: \bar{x}\left(t, t_{0}, \bar{x}\left(t_{0}\right)\right)$ only goes through the value of 0 at some time points, but doesn't remain this value as $t \in\left[t_{1}^{*}, t_{1}^{*}+T_{1}\right]$.

Let $\left\{t_{i}: i \in \ell\right\} \subset\left[t_{1}^{*}, t_{1}^{*}+T_{1}\right]$ be a time sequence that $\bar{x}\left(t, t_{0}, \bar{x}\left(t_{0}\right)\right)$ passes through 0 at each time point $t_{i}$, where $\ell$ is an index set. For convenience, we assume that $t_{i}<t_{i+1}, i, i+1 \in \ell$. Note that $\left\{t_{i}: i \in \ell\right\}$ must be a finite set. If not, a infinite subsequence $\left\{t_{i_{n}}: i_{n} \in \ell\right\}$ of $\left\{t_{i}: i \in \ell\right\}$ would exist such that $\lim _{n \rightarrow \infty} t_{i_{n}}=t^{*}, t^{*} \in\left[t_{1}^{*}, t_{1}^{*}+T_{1}\right]$. Then, there exists $n^{*}>0$ such 
that as $n>n^{*},\left|t_{i_{n}}-t^{*}\right|<\epsilon$ for any $\epsilon>0$. Hence, for any $t \in\left(t^{*}-\epsilon, t^{*}\right)$, it follows $\bar{x}\left(t, t_{0}, \bar{x}\left(t_{0}\right)\right) \equiv 0$ on account of the continuity of $\bar{x}\left(t, t_{0}, \bar{x}\left(t_{0}\right)\right)$ and $\dot{\bar{x}}\left(t, t_{0}, \bar{x}\left(t_{0}\right)\right)$. This contradicts Case 2 . Based on the above analysis, let $\left\{t_{i}\right\}_{i=1}^{n}$ be a finite set that $\bar{x}\left(t, t_{0}, \bar{x}\left(t_{0}\right)\right)$ passes through 0 at each $t_{i}$. There exists $\epsilon_{1}>0$ such that set $\Upsilon=\bigcup_{i=1}^{n}\left(t_{i}-\epsilon_{0}, t_{i}+\epsilon_{0}\right)$ is nonempty open set for any $\epsilon_{0}>0,0<\epsilon_{0}<\epsilon_{1}$. Then, on the compact set $\left[t_{1}^{*}, t_{1}^{*}+T_{1}\right] \backslash \Upsilon$, $\bar{x}\left(t, t_{0}, \bar{x}\left(t_{0}\right)\right) \neq 0$. Since the continuity of $\bar{x}\left(t, t_{0}, \bar{x}\left(t_{0}\right)\right)$ on $\left[t_{1}^{*}, t_{1}^{*}+T_{1}\right] \backslash \Upsilon$, a constant $l_{4}>0$ exists here such that $V \geq l_{4}, \forall t \in\left[t_{1}^{*}, t_{1}^{*}+T_{1}\right] \backslash \Upsilon$.

From (24), we obtain that there exists $\tilde{k}>1$, such that for $k>\tilde{k}$,

$$
\frac{1}{k^{2}} \bar{\Theta}^{T} \bar{\Theta}<l_{4} \leq V, \quad \forall t \in\left[t_{1}^{*}, t_{1}^{*}+T_{1}\right] \backslash \Upsilon
$$

and

$$
\frac{(2 k)^{\frac{\alpha}{2}} \lambda_{\min }^{\frac{3+2 \alpha}{2}}(M)}{2 N^{\alpha} \lambda_{\Phi} \lambda_{\max }^{\frac{1+\alpha}{2}}(M)}>1,
$$

with $\lambda_{\Phi}>0$ based on the fact that $\Phi M^{T} M \Phi^{T}<\lambda_{\Phi}^{2} I$ due to $\Phi$ being uniformly bounded. From (26), (29), (30) and noting that $V \geq \frac{1}{2 k} \lambda_{\min }(M) \bar{x}^{T} \bar{x}$, we obtain that

$$
\begin{aligned}
\dot{V} & <-l_{2} V^{\frac{1+\alpha}{2}}+\frac{\lambda_{\Phi}}{k}\left(\bar{x}^{T} \bar{x}\right)^{\frac{1}{2}}\left(\bar{\Theta}^{T} \bar{\Theta}\right)^{\frac{1}{2}} \\
& <-l_{2} V^{\frac{1+\alpha}{2}}+\frac{(2 k)^{\frac{1}{2}} \lambda_{\Phi}}{\lambda_{\min }^{\frac{1}{2}}(M)} V \\
& <-\frac{1}{2} l_{2} V^{\frac{1+\alpha}{2}}, \quad \forall t \in\left[t_{1}^{*}+T_{0}, t_{1}^{*}+T_{1}\right],
\end{aligned}
$$

with $0<T_{0}<T_{1}$. Due to Lemma 2 , this error system is finite-time stable and the settling time of it satisfies

$$
\begin{aligned}
T_{s} & <\frac{2^{\frac{3-\alpha}{2}} N^{\alpha} \lambda_{\max }^{\frac{1+\alpha}{2}}(M)}{(1-\alpha) k^{\frac{1+\alpha}{2}} \lambda_{\min }^{1+\alpha}(M)} V^{\frac{1-\alpha}{2}} \\
& <\frac{2^{\frac{3-\alpha}{2}} N^{\alpha} \lambda_{\max }^{\frac{1+\alpha}{2}}(M)}{(1-\alpha) k^{\frac{1+\alpha}{2}} \lambda_{\min }^{1+\alpha}(M)} .
\end{aligned}
$$

This implies $\bar{x}\left(t, t_{0}, \bar{x}\left(t_{0}\right)\right)=0$ for any $t \in\left[t_{1}^{*}+T_{0}, t_{1}^{*}+T_{1}\right]$, which is not in accord with our assumption of this case. Therefore, the second case does not exist. 
Case $3: \bar{x}\left(t, t_{0}, \bar{x}\left(t_{0}\right)\right) \neq 0$ for any $t \in\left[t_{1}^{*}, t_{1}^{*}+T_{1}\right]$.

Employing the approach mentioned above, it can also be derived that the last case does not exist.

In conclusion, there exist $k>\tilde{k}, 0<\epsilon_{0}<\epsilon_{1}$ such that, for any $\alpha \in\left(\varepsilon_{1}, 1\right)$, the error system (13)-(14) is finite-time stable on $\Omega_{1}$ and the settling time $T_{s}<\frac{2^{(3-\alpha) / 2} N^{\alpha} \lambda_{\max }^{(1+\alpha) / 2}(M)}{(1-\alpha) k^{(1+\alpha) / 2} \lambda_{\min }^{1+\alpha}(M)}$.

Remark 8. Parameters $k_{1}$ and $k_{2}$ are chosen according to the practical demand. Parameter $k$ is determined by the inequalities (29) and (30).

Proof of Theorem 1. Applying Lemma 3 and 4, we can easily derive Theorem 1.

\subsection{Decentralized finite-time adaptive consensus with directed switching topolo- gies}

Decentralized finite-time adaptive consensus problem for the leader-following multiagent system (1)-(2), whose network topology is directed and switching while satisfying detailed balance condition, will be discussed in this part.

Note that the network topology of the system is time variant throughout the system time. A bounded contiguous time sequence $\left[t_{k}, t_{k+1}\right), k=$ $0,1,2, \ldots$, with $t_{0}=0, \mathscr{T}_{0} \leq t_{k+1}-t_{k} \leq \mathscr{T}$ for two positive constants $\mathscr{T}_{0}$ and $\mathscr{T}$, is introduced here. Suppose that time varying network topology switches at time points $t_{k}, k=0,1,2, \ldots$, and is unchanged in each time interval $\left[t_{k}, t_{k+1}\right)$. The notations $\left\{\overline{\mathscr{G}}_{s} \mid s \in \mathscr{S}\right\}$ and $\left\{\mathscr{G}_{s} \mid s \in \mathscr{S}\right\}$ are introduced here to respectively denote all possible graphs on node set $\overline{\mathscr{V}}$ and corresponding subgraphs on node set $\mathscr{V}$ with $\mathscr{S}$ being a finite index set. For convenience's sake, a piecewise constant switching signal $\sigma(t):[0, \infty) \rightarrow \mathscr{S}$ is defined here to to depict the time dependence of graphs. Then, the possible graphs at time $t$ on node set $\overline{\mathscr{V}}$ and node set $\mathscr{V}$ can be respectively denoted as $\overline{\mathscr{G}}_{\sigma(t)}$ and $\mathscr{G}_{\sigma(t)}$. Notations $\mathscr{N}_{i}(t), a_{i j}(t), b_{i}(t)$ and $\mathscr{L}_{\sigma(t)}$ are used here to describe the time varying versions of neighbor sets $\mathscr{N}_{i}$ of all follower agents, all $i j$ th items $a_{i j}$ of adjacency matrix $\mathscr{A}$, all weighting coefficients $b_{i}$, and Laplacian matrix $\mathscr{L}$, respectively. Throughout this paper, signal $\sigma(t)$ is assumed to switch finite times in any bounded time interval.

Assumption 2. The directed graph $\mathscr{G}_{\sigma(t)}$ is strongly connected and detailed balanced, and $b_{i}(t)>0$ for at least one $i \in \mathscr{V}$. 
For directed switching network topologies, decentralized finite-time adaptive consensus control schemes proposed here can be split into the following two parts.

Decentralized finite-time feedback laws:

$$
\begin{aligned}
u_{i}= & -k\left[\omega _ { i } ( t ) \left[\sum_{j \in \mathscr{N}_{i}(t)} a_{i j}(t)\left(x_{i}-x_{j}\right)\right.\right. \\
& \left.\left.+b_{i}(t)\left(x_{i}-x_{0}\right)\right]\right]^{\alpha}+\Phi_{i}^{T} \hat{\Theta}_{i},
\end{aligned}
$$

and decentralized finite-time parameter adaptive laws:

$$
\begin{aligned}
\dot{\hat{\theta}}_{0 i}= & -\frac{k_{1}}{k} \phi_{0}(t) \omega_{i}(t) \times \\
& {\left[\sum_{j \in \mathscr{N}_{i}(t)} a_{i j}(t)\left(x_{i}-x_{j}\right)+b_{i}(t)\left(x_{i}-x_{0}\right)\right], } \\
\dot{\hat{\theta}}_{i}= & \frac{k_{2}}{k} \phi_{i}\left(x_{i}, t\right) \omega_{i}(t) \times \\
& {\left[\sum_{j \in \mathscr{N}_{i}(t)} a_{i j}(t)\left(x_{i}-x_{j}\right)+b_{i}(t)\left(x_{i}-x_{0}\right)\right], }
\end{aligned}
$$

where $i \in \mathscr{V}$.

Letting $M_{\sigma(t)}=\operatorname{diag}\{\omega\}_{\sigma(t)} H_{\sigma(t)}, \eta=\operatorname{col}\left(\eta_{1}, \eta_{2}, \ldots, \eta_{N}\right)=M_{\sigma(t)} \bar{x}$, the error system for the case of directed switching network topologies can be written as follows:

$$
\dot{\bar{x}}(t)=-k\lceil\eta\rfloor^{\alpha}+\Phi^{T} \bar{\Theta}
$$

and

$$
\dot{\bar{\Theta}}=-\frac{1}{k}\left(I_{N} \otimes K\right) \Phi M_{\sigma(t)} \bar{x} .
$$

For each $s \in \mathscr{S}$, define $\mu_{s}=\lambda_{\min }\left(M_{s}\right)$ and $\nu_{s}=\lambda_{\max }\left(M_{s}\right)$. Depending on Lemma 1 , it follows that

$$
\delta_{\min }=\min \left\{\mu_{s} \mid s \in \mathscr{S}\right\}, \delta_{\max }=\max \left\{\nu_{s} \mid s \in \mathscr{S}\right\}
$$

are positive and not dependent on time $t$.

For the system (1)-(2) under the case of directed switching network topologies, another main theorem can be obtained. 
Theorem 2. Consider the multiagent system (1)-(2). Suppose that Assumption 2 holds, $\phi_{i}(t), i \in \overline{\mathscr{V}}$, are uniformly bounded and continuous, and the PE condition given in ( 7$)$ is met. Then, there exists $\varepsilon_{2} \in(0,1)$, such that, for every $\alpha \in\left(1-\varepsilon_{2}, 1\right)$, under feedback laws (33) and parameter adaptive laws (34), we have: (a) global consensus for this system is attained in finite-time; (b) global parameter convergence is assured in finite-time; (c) the settling time $T_{s}<\frac{2^{(3-\alpha) / 2} N^{\alpha} \delta_{\max }^{(1+\alpha) / 2}}{(1-\alpha) k^{(1+\alpha) / 2} \delta_{\min }^{1+\alpha}}$.

Proof. The proof of this theorem is similar to that of Theorem 1.

\section{Simulations}

To validate the proposed decentralized finite-time adaptive consensus schemes, an example of a system consisting of 5 follower agents and 1 leader agent, whose network topology is directed and switching while satisfying detailed balance condition, is given in this section. The dynamics of follower agents are modeled as

$$
\begin{aligned}
f_{i}\left(x_{i}(t), t\right) & =\frac{i}{2} \sin t+\exp \left(\bar{x}_{i}\right) \\
& =\left(\sin t, \exp \left(\bar{x}_{i}\right)\right)\left(\begin{array}{c}
\frac{i}{2} \\
1
\end{array}\right) \\
& =\phi_{i}^{T}\left(x_{i}(t), t\right) \theta_{i}
\end{aligned}
$$

with $\phi_{i}=\left(\begin{array}{c}\sin t \\ \exp \left(\bar{x}_{i}\right)\end{array}\right), \theta_{i}=\left(\begin{array}{c}\frac{i}{2} \\ 1\end{array}\right), i=1,2,3,4,5$, and the leader agent described by

$$
\dot{x}_{0}(t)=\frac{\sqrt{3}}{4} \cos t
$$

where control input $v_{0}(t)=\frac{\sqrt{3}}{4} \cos t=\phi_{0}^{T}(t) \theta_{0}$ with $\phi_{0}=$ cost, $\theta_{0}=\frac{\sqrt{3}}{4}$.

In this example, a finite set of graphs $\left\{G_{1}, G_{2}, G_{3}, G_{4}\right\}$ described in Fig.2 shows different underlying network topologies of the system considered in this part. The network topology of this system automatically switches every four time steps in the following way: $G_{1} \rightarrow G_{2} \rightarrow G_{3} \rightarrow G_{4} \rightarrow G_{1} \rightarrow \cdots$, to the next graph. In the Fig. 2, the numbers next to the edges are the 
coupling weights, which are chosen in $[0.5,1.5]$ randomly. In order to ensure that the above four graphs are detailed balanced, we select

$$
\begin{aligned}
& \operatorname{diag}\{\omega\}_{G_{1}}=\operatorname{diag}\{15 / 7,1,3 / 2,81 / 182,9 / 13\}, \\
& \operatorname{diag}\{\omega\}_{G_{2}}=\operatorname{diag}\{1,9 / 11,6 / 11,144 / 91,12 / 13\}, \\
& \operatorname{diag}\{\omega\}_{G_{3}}=\operatorname{diag}\{1,3 / 2,35 / 11,15 / 11,1\}, \\
& \operatorname{diag}\{\omega\}_{G_{4}}=\operatorname{diag}\{1,5 / 3,3 / 4,26 / 9,13 / 7\},
\end{aligned}
$$

respectively. By a straightforward calculation, the smallest nonzero eigenvalue $\delta_{\min }$ and biggest eigenvalue $\delta_{\max }$ of $M_{\sigma(t)}=\operatorname{diag}\{\omega\}_{\sigma(t)} H_{\sigma(t)}$ are respectively 0.1253 and 6.3524 .
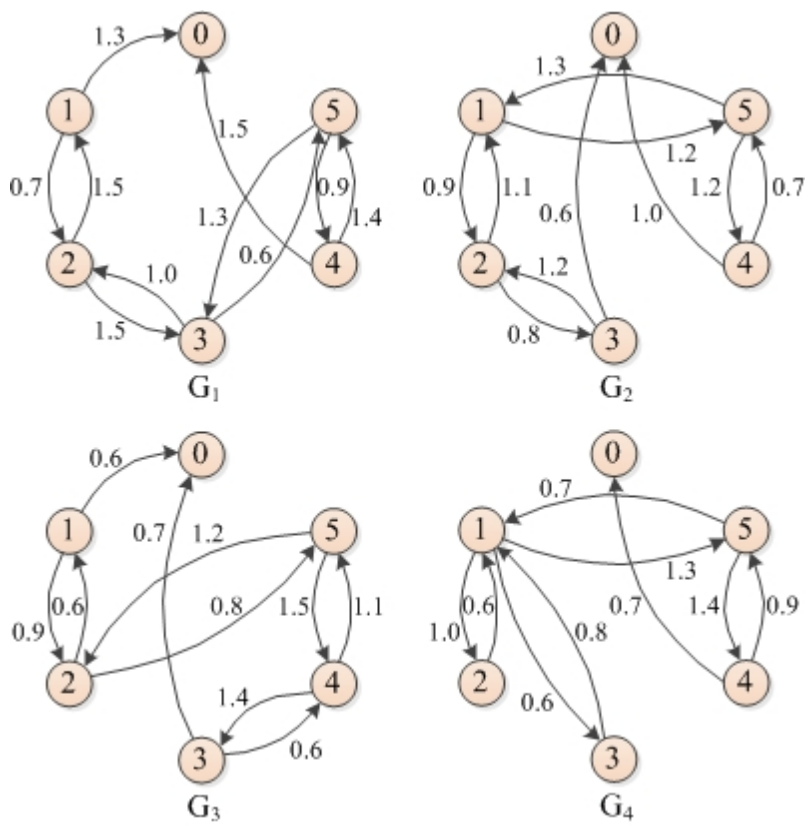

Figure 2: Switching graphs

For the system (35)-(36), all the agents are static at $t=0$. The initial consensus error vector is $\bar{x}(0)=(-3.9,4.6,-4.9,2.7,3.2)^{T}$ and vector $\bar{\Theta}(0)=$ $(-2.5,-2.7,0.2,1.7,2.6,-2.2,0.4,-0.2,-2.9,-1.0,-2.0,1.8,-1.1,0.2,-2.0)^{T}$ is the initial parameter estimate error. Under decentralized feedback laws (33) and decentralized parameter adaptive laws (34) with $\alpha=0.7, k=2.0$, $k_{1}=0.7, k_{2}=0.7$, simulation is conducted in $300 \mathrm{~s}$. By simple computation, we get $T_{s}<2082.4 s$. Let $\lambda_{P E}(t)$ be the minimum eigenvalue of $\int_{t}^{t+\delta_{0}} \Phi \Phi^{T} d \tau$. 


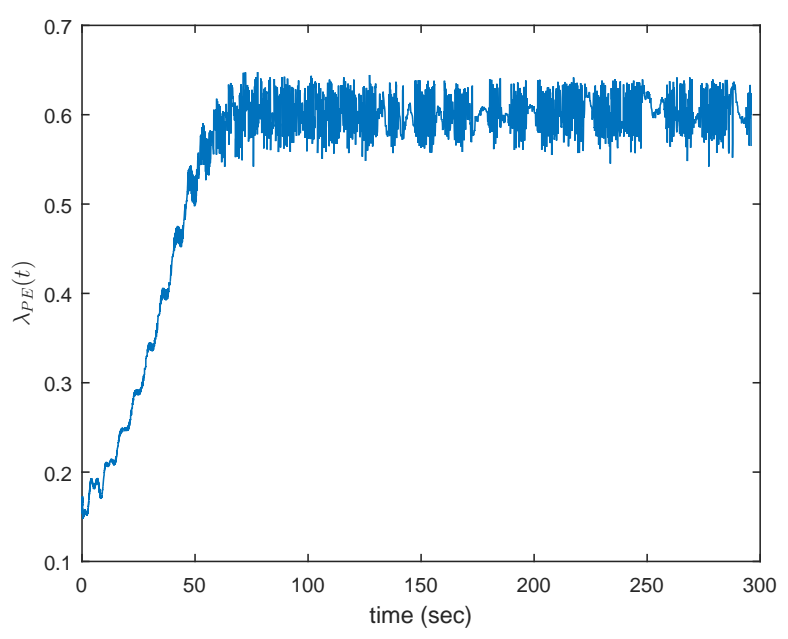

Figure 3: The PE condition is satisfied.

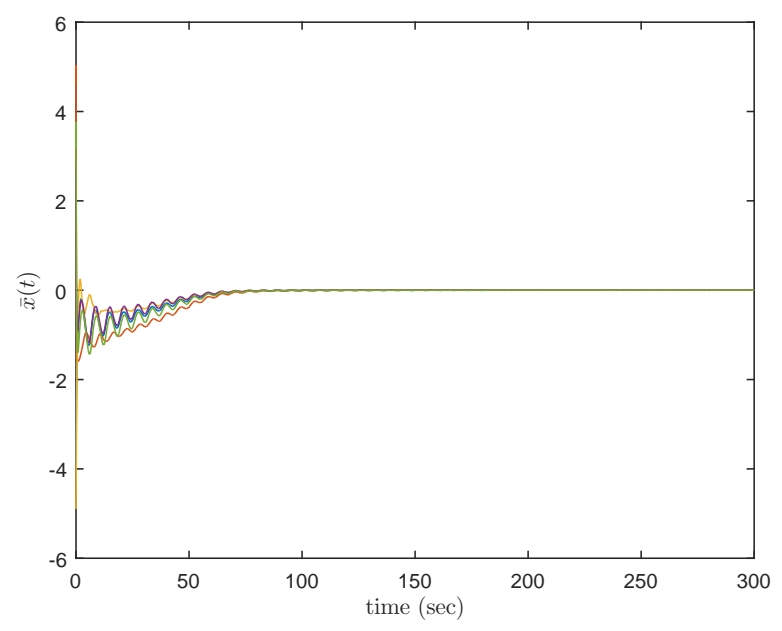

Figure 4: Five components of consensus error vector $\bar{x}(t)$.

By choosing $\delta_{0}=4$, Fig. 3 shows that $\lambda_{P E}(t)>0$ for all $t \geq 0$. From Fig. 3, we can obtain that the smallest value of $\lambda_{P E}$ is $l_{0}=0.1476$, which shows that the PE condition (7) is satisfied. Five components of the vector $\bar{x}(t)=x(t)-\mathbf{1}_{N} \otimes x_{0}(t)$ are shown in Fig. 4. The parameter estimate errors $\hat{\theta}_{0 i}-\theta_{0}$ and $\hat{\theta}_{i}-\theta_{i}, i=1,2,3,4,5$, are shown in Figs. 5 and 6 , respectively. As illustrated in Fig. 4, all follower agents follow the leader agent in finite- 


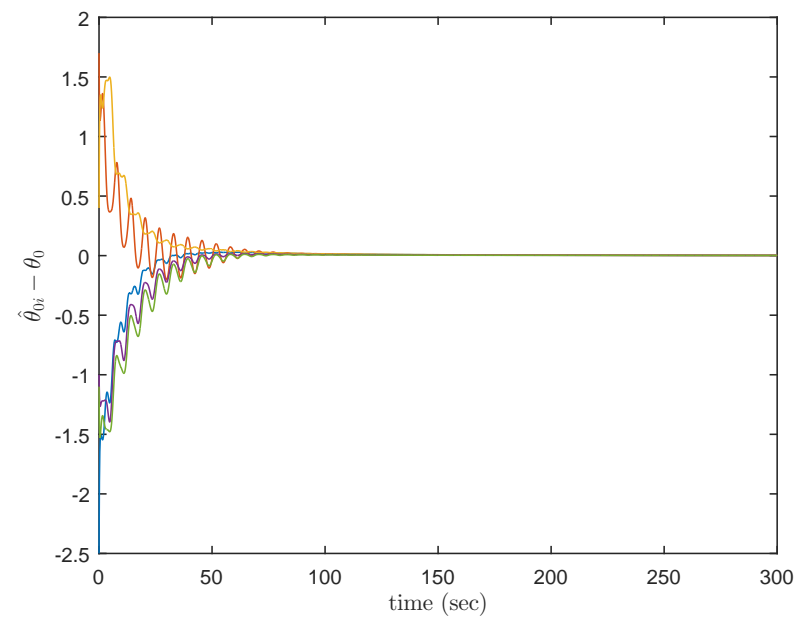

Figure 5: Parameter estimate error $\hat{\theta}_{0 i}-\theta_{0}, i=1,2,3,4,5$.

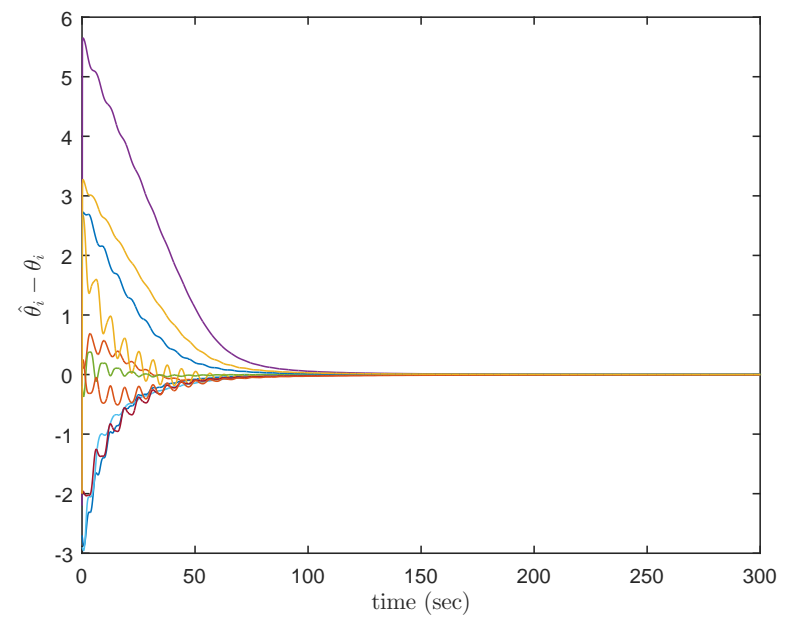

Figure 6: Parameter estimate error $\hat{\theta}_{i}-\theta_{i}, i=1,2,3,4,5$.

time. Figs. 5 and 6 demonstrate that the parameters can be converged in finite-time. 


\section{Conclusions}

In this paper, finite-time adaptive consensus problem was researched for first-order multiagent systems with unknown nonlinear dynamics. Linearly parameterized method was introduced to model unknown nonlinear dynamics of the systems. By only utilizing the local relative position state information between each agent and its neighbors, decentralized finite-time adaptive consensus algorithms were presented with directed fixed and switching network topologies which satisfy detailed balance condition. Based on classical Lyapunov analysis techniques, both finite-time stability and finite-time parameter convergence were assured by employing the proposed control algorithms. Finally, the results in Simulations part were presented to validate our main results.

\section{Acknowledgements}

This work is supported in part by National Natural Science Foundation (NNSF) of China (61273183, 61174216, 61374028 and 61304162).

\section{References}

[1] A. Jadbabaie, J. Lin, A. S. Morse, Coordination of groups of mobile autonomous agents using nearest neighbor rules, IEEE Trans. Autom. Control 48 (2003) 988-1001.

[2] J. A. Fax, R. M. Murray, Information flow and cooperative control of vehicle formations, IEEE Trans. Autom. Control 49 (2004) 1465-1476.

[3] L. Moreau, Stability of multiagent systems with time-dependent communication links, IEEE Trans. Autom. Control 50 (2005) 169-182.

[4] F. Xiao, L. Wang, J. Chen, Y. Gao, Finite-time formation control for multi-agent systems, Automatica 45 (2009) 2605-2611.

[5] H. Liu, G. Xie, L. Wang, Necessary and sufficient conditions for containment control of networked multi-agent systems, Automatica 48 (2012) $1415-1422$.

[6] X. Wang, S. Li, P. Shi, Distributed finite-time containment control for double-integrator multiagent systems, IEEE Trans. Cybern. 44 (2014) $1518-1528$. 
[7] C. Wen, F. Liu, Q. Song, X. Feng, Observer-based consensus of secondorder multi-agent systems without velocity measurements, Neurocomputing 179 (2016) 298-306.

[8] R. Olfati-Saber, R. M. Murray, Consensus problems in networks of agents with switching topology and time-delays, IEEE Trans. Autom. Control 49 (2004) 1520-1533.

[9] W. Ren, R. W. Beard, Consensus seeking in multiagent systems under dynamically changing interaction topologies, IEEE Trans. Autom. Control 50 (2005) 655-661.

[10] W. Ren, Multi-vehicle consensus with a time-varying reference state, Syst. Control Lett. 56 (2007) 474-483.

[11] Y. Sun, L. Wang, Consensus of multi-agent systems in directed networks with nonuniform time-varying delays, IEEE Trans. Autom. Control 54 (2009) 1607-1613.

[12] Y. Zheng, Y. Zhu, L. Wang, Consensus of heterogeneous multi-agent systems, IET Control Theory Appl. 5 (2011) 1881-1888.

[13] H. Yu, X. Xia, Adaptive consensus of multi-agent networks with jointly connected topologies, Automatica 48 (2012) 1783-1790.

[14] G. Miao, Q. Ma, Group consensus of the first-order multi-agent systems with nonlinear input constraints, Neurocomputing 161 (2015) 113-119.

[15] J. Cortés, Finite-time convergent gradient flows with applications to network consensus, Automatica 42 (2006) 1993-2000.

[16] Q. Hui, W. M. Haddad, S. P. Bhat, Finite-time semistability and consensus for nonlinear dynamical networks, IEEE Trans. Autom. Control 53 (2008) 1887-1900.

[17] Y. Zhao, Z. Duan, G. Wen, Y. Zhang, Distributed finite-time tracking control for multi-agent systems: An observer-based approach, Syst. Control Lett. 62 (2013) 22-28.

[18] S. Yu, X. Long, Finite-time consensus for second-order multi-agent systems with disturbances by integral sliding mode, Automatica 54 (2015) 158-165. 
[19] H. Du, G. Wen, X. Yu, S. Li, M. Z. Q. Chen, Finite-time consensus of multiple nonholonomic chained-form systems based on recursive distributed observer, Automatica 62 (2015) 236-242.

[20] F. Jiang, L. Wang, Finite-time weighted average consensus with respect to a monotonic function and its application, Syst. Control Lett. 60 (2011) 718-725.

[21] S. Li, H. Du, X. Lin, Finite-time consensus algorithm for multi-agent systems with double-integrator dynamics, Automatica 47 (2011) 17061712 .

[22] Y. Zheng, L. Wang, Finite-time consensus of heterogeneous multi-agent systems with and without velocity measurements, Syst. Control Lett. 61 (2012) 871-878.

[23] X. Liu, J. Lam, W. Yu, G. Chen, Finite-time consensus of multiagent systems with a switching protocol, IEEE Trans. Neural Netw. Learn. Syst. 27 (2016) 853-862.

[24] J. Huang, C. Wen, W. Wang, Y. Song, Adaptive finite-time consensus control of a group of uncertain nonlinear mechanical systems, Automatica 51 (2015) 292-301.

[25] H. Yu, Y. Shen, X. Xia, Adaptive finite-time consensus in multi-agent networks, Syst. Control Lett. 62 (2013) 880-889.

[26] C. D. Godsil, G. Royle, Algebraic Graph Theory, Springer, New York, 2001.

[27] F. Jiang, L. Wang, Finite-time information consensus for multi-agent systems with fixed and switching topologies, Phys. D 238 (2009) 15501560 .

[28] Z. Meng, Z. Zhao, Z. Lin, On global leader-following consensus of identical linear dynamic systems subject to actuator saturation, Syst. Control Lett. 62 (2013) 132-142.

[29] S. P. Bhat, D. S. Bernstein, Finite-time stability of continuous autonomous systems, SIAM J. Control Optim. 38 (2000) 751-766. 
[30] S. Sastry, M. Bodson, Adaptive Control: Stability, Convergence, and Robustness, Prentice-Hall, New Jersey, 1989.

[31] R. Marino, P. Tomei, Nonlinear Control Design-Geometric, Adaptive and Robust, Prentice-Hall, Europe, 1995.

[32] H. Bai, M. Arcak, J. T. Wen, Adaptive motion coordination: using relative velocity feedback to track a reference velocity, Automatica 45 (2009) 1020-1025.

[33] Y. Cao, W. Ren, Finite-time consensus for multi-agent networks with unknown inherent nonlinear dynamics, Automatica 50 (2014) 26482656.

[34] H. Du, Y. He, Y. Cheng, Finite-time synchronization of a class of second-order nonlinear multi-agent systems using output feedback control, IEEE Trans. Circuits Syst. I 61 (2014) 1778-1788.

[35] Y. Zhao, Z. Duan, G. Wen, G. Chen, Distributed finite-time tracking of multiple non-identical second-order nonlinear systems with settling time estimation, Automatica 64 (2016) 86-93.

[36] Y. Wang, Y. Song, M. Krstic, C. Wen, Fault-tolerant finite time consensus for multiple uncertain nonlinear mechanical systems under singleway directed communication interactions and actuation failures, Automatica 63 (2016) 374-383.

[37] H. Eisenbeiss, A mini unmanned aerial vehicle (UAV): system overview and image acquisition, in: International Workshop on Processing and Visualization Using High-Resolution Imagery.

[38] J. F. Roberts, T. Stirling, J. C. Zufferey, D. Floreano, 3-D relative positioning sensor for indoor flying robots, Auton. Robot. 33 (2012) $5-20$.

[39] S. Li, X. Wang, Finite-time consensus and collision avoidance control algorithms for multiple AUVs, Automatica 49 (2013) 3359-3367. 